\title{
On Integrable Perturbations of Some Nonholonomic Systems ${ }^{\star}$
}

Andrey V. TSIGANOV ${ }^{\dagger \ddagger}$

$\dagger$ St. Petersburg State University, St. Petersburg, Russia

E-mail: andrey.tsiganov@gmail.com

$\dagger$ Udmurt State University, 1 Universitetskaya Str., Izhevsk, Russia

Received May 08, 2015, in final form October 16, 2015; Published online October 20, 2015

http://dx.doi.org/10.3842/SIGMA.2015.085

\begin{abstract}
Integrable perturbations of the nonholonomic Suslov, Veselova, Chaplygin and Heisenberg problems are discussed in the framework of the classical Bertrand-Darboux method. We study the relations between the Bertrand-Darboux type equations, well studied in the holonomic case, with their nonholonomic counterparts and apply the results to the construction of nonholonomic integrable potentials from the known potentials in the holonomic case.
\end{abstract}

Key words: nonholonomic system; integrable systems

2010 Mathematics Subject Classification: 37J60; 70G45; 70H45

Dedicated to Sergio Benenti on the occasion of his 70th birthday

\section{Introduction}

In classical mechanics, the Euler-Poisson equations

$$
\mathbf{I} \dot{\omega}=\mathbf{I} \omega \times \omega+\gamma \times \frac{\partial V(\gamma)}{\partial \gamma}, \quad \dot{\gamma}=\gamma \times \omega
$$

describe the rotation of a rigid body with a fixed point using a rotating reference frame with its axes fixed in the body and parallel to the body's principal axes of inertia. Here $\omega=\left(\omega_{1}, \omega_{2}, \omega_{3}\right)$ is the angular velocity vector of the $\operatorname{body}, \mathbf{I}=\operatorname{diag}\left(I_{1}, I_{2}, I_{3}\right)$ is a tensor of inertia, $\gamma=\left(\gamma_{1}, \gamma_{2}, \gamma_{3}\right)$ is a unit Poisson vector and $V(\gamma)$ is a potential field. All the vectors are expressed in the so-called body frame and $x \times y$ means the cross product of two vectors in three-dimensional Euclidean space.

Let us impose a nonholonomic constraint on the angular velocity

$$
f=(\omega, a)=0 \quad \text { or } \quad f=(\omega, \gamma)=0,
$$

where $a$ is a fixed unit vector in the rotating frame for the Suslov problem [33], $\gamma$ is a fixed unit vector in the stationary frame for the Veselova problem [40] and $(x, y)$ means the scalar product of two vectors. In this case the Euler-Poisson equations (1.1) are replaced by equations

$$
\mathbf{I} \dot{\omega}=\mathbf{I} \times \omega+\gamma \times \frac{\partial V(\gamma)}{\partial \gamma}+\lambda n, \quad \dot{\gamma}=\gamma \times \omega, \quad n=a, \gamma,
$$

where $\lambda$ is a Lagrange multiplier which has to be found from the condition $\dot{f}=0$.

\footnotetext{
${ }^{\star}$ This paper is a contribution to the Special Issue on Analytical Mechanics and Differential Geometry in honour of Sergio Benenti. The full collection is available at http://www.emis.de/journals/SIGMA/Benenti.html
} 
Both systems of differential equations (1.1) and (1.2) are geometrically interpreted in terms of the vector field $X$

$$
\dot{x}_{i}=X_{i}\left(x_{1}, \ldots, x_{6}\right)
$$

in a six-dimensional manifold $M$ with coordinates $x=(\omega, \gamma)$. The classical Euler-Jacobi theorem says that the vector field $X(1.3)$ on a six-dimensional manifold $M$ is integrable by quadratures if it has an invariant volume form (invariant measure) and four functionally independent first integrals $[28,37]$.

Equations (1.1) and (1.2) preserve the norm of the unit Poisson vector $\gamma$

$$
C_{1}=(\gamma, \gamma)=\gamma_{1}^{2}+\gamma_{2}^{2}+\gamma_{3}^{2}=1,
$$

and mechanical energy

$$
H_{1}=\frac{1}{2}(I \omega, \omega)+\frac{1}{2} V(\gamma)
$$

An additive perturbations (1.2) of the Euler-Poisson vector field (1.1) change the standard invariant volume form and the second geometric first integral

$$
C_{2}=(\omega, \mathbf{I} \gamma) \rightarrow C_{2}=(\omega, n),
$$

see details in [15]. Thus, according to the Euler-Jacobi theorem equations (1.1) and (1.2) are integrable by quadratures if there is one more independent first integral $\mathrm{H}_{2}$.

There are several methods to uncover integrals of motion. A one of the simplest method considers function $H_{2}$ polynomial in the velocities, with the coefficients being arbitrary functions of the coordinates, and requires that it is conserved in time

$$
\dot{H}_{2}=0 \text {. }
$$

This condition yields a system of coupled partial differential equations on the coefficients, which are some of the most well-studied first-order PDE's in classical mechanics [26].

In this paper, we want to study what is going on with these well-known PDE's when we impose nonholonomic constraints on the rigid body motion. Some partial solutions of these new PDE's are discussed in literature, see, e.g., [14, 20, 29] and references within. Our main aim is to prove that these PDE's for the nonholonomic Chaplygin, Suslov and Veselova systems can be easily reduced to the well-studied PDE's for the Hamiltonian vector field (1.1). Consequently, we can directly obtain all the possible integrable perturbations of these nonholonomic systems directly from the well-known integrable potentials of the Hamiltonian mechanics.

The necessary references to the main aspects of nonholonomic mechanics can be found in several papers of Sergio Benenti dedicated to the analysis of nonholonomic mechanical systems $[3,5,6,7]$. Following in the steps of these papers we will consider nonholonomic systems using only the knowledge of the basic notions of analytical mechanics, i.e., utilizing a 'user-friendly' approach to the dynamics of nonholonomic systems proposed by Sergio Benenti [5].

This paper is organized as follows. In Section 2 we will introduce Bertarnd-Darboux equation for the holonomic particle on the plane and its counterpart for the holonomic particle on the sphere. Section 3 contains the main result of integrable perturbations for the Suslov system. We will show that integrable potentials for the nonholonomic Suslov problem satisfy to the standard Bertarnd-Darboux equation for the holonomic particle in the plane. Sections 4 and 5 are devoted to the review of the known integrable potentials for the Veselova and Chaplygin systems. We will give the Bertrand-Darboux type equations for these systems and will show how these equations are reduced to the Bertarnd-Darboux equation for the holonomic particle on the sphere. Section 6 deals with two nonholonomic systems on the plane and contains the Bertrand-Darboux type equations for the nonholonomic oscillator and the Heisenberg system (nonholonomic integrator). Finally, we briefly discuss the quasi-integrable potentials for the Suslov and Veselova problems, which were introduced by Llibre et al. 


\section{Integrable potentials on the plane and sphere}

In [9] Bertrand studied the Newton equations for a particle on the plane

$$
\frac{d^{2} q_{1}}{d t^{2}}=F_{1}, \quad \frac{d^{2} q_{2}}{d t^{2}}=F_{2}, \quad F_{k}=-\frac{\partial V\left(q_{1}, q_{2}\right)}{\partial q_{k}}
$$

and tried to solve equation (1.4) using linear, quadratic or fractional (linear/linear) anzats in the velocity for the additional integral of motion.

In particular, according to Bertrand [9], if

$$
H_{2}=\sum_{i, j=1}^{2} K_{i j}\left(q_{1}, q_{2}\right) \dot{q}_{i} \dot{q}_{j}+U\left(q_{1}, q_{2}\right),
$$

then one equation $\dot{H}_{2}=0$ yields two systems of PDE's. The generic solution of the first system of equations for the coefficients $K_{i j}\left(q_{1}, q_{2}\right)$

$$
\begin{aligned}
H_{2}= & \left(-\frac{\alpha}{2} q_{2}^{2}-\beta_{2} q_{2}+\frac{\gamma_{11}}{2}\right) p_{1}^{2}+\left(-\frac{\alpha}{2} q_{1}^{2}-\beta_{1} q_{1}+\frac{\gamma_{22}}{2}\right) p_{2} \\
& +\left(\alpha q_{1} q_{2}+\beta_{1} q_{2}+\beta_{2} q_{1}+\gamma_{12}\right) p_{1} p_{2}+U(q)
\end{aligned}
$$

depends on the six constants of integration $\alpha, \beta_{1}, \beta_{2}, \gamma_{11}, \gamma_{12}, \gamma_{22}$.

In order to describe the forces $F_{1,2}$ that should act on the particle Bertrand also extracted one linear second-order PDE on potential $V$ from the coupled system of equations on the potentials $V$ and $U$

$$
\begin{aligned}
& \left(\alpha q_{1} q_{2}+\beta_{1} q_{1}+\beta_{2} q_{2}+\gamma_{12}\right)\left(\partial_{22} V-\partial_{11} V\right) \\
& \quad+\left(\alpha q_{1}^{2}-\alpha q_{2}^{2}+2 \beta_{1} q_{1}-2 \beta_{2} q_{2}+\gamma_{11}-\gamma_{22}\right) \partial_{12} V \\
& \quad+3\left(\alpha q_{1}+\beta_{1}\right) \partial_{2} V-3\left(\alpha q_{2}+\beta_{2}\right) \partial_{1} V=0,
\end{aligned}
$$

where $\partial_{i}=\partial / \partial q_{i}$ and $\partial_{i k}=\partial^{2} / \partial q_{i} \partial q_{k}$.

In [9] Bertand studied only some partial solutions of this equation, whereas in [17] Darboux gave a complete solution and, therefore, now equation (2.2) is called the Bertrand-Darboux equation. Later on Darboux results were included almost verbatim in the classical text of Whittaker on analytical mechanics, see historical details in [32].

Ideas used by Bertarnd and Darboux to solve the Bertrand-Darboux problem were generalized to study Hamiltonian systems defined in Euclidean spaces of higher dimensions and in other (pseudo) Riemannian manifolds. According to Eisenhard [21], the first system of PDE's is the Killing equation for the Killing tensor of second order with vanishing Haantjes torsion, hereafter called the characteristic Killing tensor on the Riemannian manifold $Q$. The second system of PDE's on the various Riemannian manifolds was studied by Kalnins, Miller [25], Benenti [2, 4] etc.

In particular, Benenti formulated the following proposition.

Proposition 1. A natural Hamiltonian $H=\sum \mathrm{g}_{i j} p_{i} p_{j}+V$ on the cotangent bundle $T^{*} Q$ of a Riemannian manifold $Q$ is separable in orthogonal coordinates iff on $Q$ there exists a Killing $K$ of second order with simple eigenvalues and normal eigenvectors, so that

$$
\mathrm{d}(K \mathrm{~d} V)=0 .
$$

Separable Hamiltonian flow has a necessary number of first integrals, which can be directly calculated from the characteristic Killing tensor $K$, which satisfies to the Killing equation

$$
\nabla_{\alpha} K_{\beta \gamma}+\nabla_{\beta} K_{\gamma \alpha}+\nabla_{\gamma} K_{\alpha \beta}=0
$$


where $\nabla$ is the Levi-Civita connection of the Riemannian metric [2, 4]. Tensor $K$ has normal eigenvectors if and only if its Haantjes torsion is equal to zero. Integrable systems associated with the Killing tensors of second order with nontrivial Haantjes torsion were found only recently [38].

\subsection{Bertrand-Darboux type equation on the sphere}

Let us consider the standard Hamiltonian vector field describing rotation of a rigid body fixed at the point

$$
\dot{M}=M \times \omega, \quad \dot{\gamma}=\gamma \times \omega .
$$

Here $M$ is the angular momentum, $\omega=\mathbf{A} M$ is the angular velocity, $\gamma$ is the constant unit vector in a moving frame an $\mathbf{A}$ is the diagonal inverse to $\mathbf{I}$ matrix

$$
\mathbf{A}=\mathbf{I}^{-1}=\left(\begin{array}{ccc}
a_{1} & 0 & 0 \\
0 & a_{2} & 0 \\
0 & 0 & a_{3}
\end{array}\right), \quad a_{k}=\frac{1}{I_{k}} .
$$

According to Euler, there are two first integrals of second order in momenta

$$
H_{1}=\frac{1}{2}(M, \mathbf{A} M), \quad H_{2}=(M, M)
$$

and two geometric integrals

$$
C_{1}=\gamma_{1}^{2}+\gamma_{2}^{2}+\gamma_{3}^{2}, \quad C_{2}=\gamma_{1} M_{1}+\gamma_{2} M_{2}+\gamma_{3} M_{3},
$$

which are the Casimir functions of the underlying Poisson structure.

Let us consider perturbation of the free motion (2.4) by adding forces associated with potential field $V_{1}(\gamma)$. For the perturbed Hamiltonian vector field

$$
\dot{M}=M \times \omega+\gamma \times \frac{\partial V_{1}(\gamma)}{\partial \gamma}, \quad \dot{\gamma}=\gamma \times \omega,
$$

functions $C_{1,2}$ are also constants of motion and, therefore, we can exclude the third component of the Poisson vector $\gamma$ from other calculations

$$
\gamma_{3}=\sqrt{1-\gamma_{1}^{2}-\gamma_{2}^{2}}
$$

Substituting the polynomials of second order in momenta

$$
H_{1}=\frac{1}{2}(M, \mathbf{A} M)+V_{1}(\gamma), \quad H_{2}=(M, M)+V_{2}(\gamma)
$$

in the equations $\dot{H}_{1,2}=0$ one gets three partial differential equations on $V_{1,2}(\gamma)$

$$
\begin{aligned}
& \partial_{2}\left(a_{1} V_{2}-2 V_{1}\right)=0, \quad \partial_{1}\left(a_{2} V_{2}-2 V_{1}\right)=0, \\
& a_{3}\left(\gamma_{2} \partial_{1} V_{2}-\gamma_{1} \partial_{2} V_{2}\right)+2 \gamma_{1} \partial_{2} V_{1}-2 \gamma_{2} \partial_{1} V_{1}=0 .
\end{aligned}
$$

Here $V_{1,2}(\gamma)$ are functions on two independent variables $\gamma_{1}, \gamma_{2}$ and $\partial / \partial \gamma_{k}=\partial_{k}$.

The generic solution of these equations (2.8)

$$
V_{1}=-\frac{1}{2}\left(a_{2} a_{3} \gamma_{1}^{2}+a_{1} a_{3} \gamma_{2}^{2}+a_{1} a_{2} \gamma_{3}^{2}\right), \quad V_{2}=a_{1} \gamma_{1}^{2}+a_{2} \gamma_{2}^{2}+a_{3} \gamma_{3}^{2}
$$

is associated with the Clebsh system [26]. 
At $C_{2}=(\gamma, M)=0$ the phase space is equivalent to the cotangent bundle of the twodimensional sphere [12]. In this case equation $\dot{H}_{2}=0$ yields only two equations

$$
\begin{aligned}
& \left(\gamma_{2}^{2}\left(a_{2}-a_{3}\right)-a_{2}\right) \partial_{1} V_{2}-\gamma_{1} \gamma_{2}\left(a_{1}-a_{3}\right) \partial_{2} V_{2}+2 \partial_{1} V_{1}=0, \\
& \left(\gamma_{1}^{2}\left(a_{1}-a_{3}\right)-a_{1}\right) \partial_{2} V_{2}-\gamma_{1} \gamma_{2}\left(a_{2}-a_{3}\right) \partial_{1} V_{2}+2 \partial_{2} V_{1}=0,
\end{aligned}
$$

from which we can easily get the equation on one potential. Namely, when equations are differentiated by $\gamma_{1,2}$ and subtracted from each other, the result is

$$
\begin{aligned}
& \partial_{1}\left(\left(\gamma_{2}^{2}\left(a_{2}-a_{3}\right)-a_{2}\right) \partial_{1} V_{2}-\gamma_{1} \gamma_{2}\left(a_{1}-a_{3}\right) \partial_{2} V_{2}\right) \\
& \quad-\partial_{2}\left(\left(\gamma_{1}^{2}\left(a_{1}-a_{3}\right)-a_{1}\right) \partial_{2} V_{2}-\gamma_{1} \gamma_{2}\left(a_{2}-a_{3}\right) \partial_{1} V_{2}\right)=0 .
\end{aligned}
$$

It is well-known that the characteristic equation (2.3), (2.10) has a continuum of solutions labelled by two arbitrary functions $G_{1,2}$

$$
V_{1}=\frac{u_{2} G_{1}\left(u_{1}\right)-u_{1} G_{2}\left(u_{2}\right)}{2\left(u_{2}-u_{1}\right)}, \quad V_{2}=\frac{G_{1}\left(u_{1}\right)-G_{2}\left(u_{2}\right)}{u_{2}-u_{1}} .
$$

Here $u_{1}, u_{2}$ are sphero-conical coordinates on the sphere

$$
\gamma_{i}=\sqrt{\frac{\left(u_{1}-a_{i}\right)\left(u_{2}-a_{i}\right)}{\left(a_{j}-a_{i}\right)\left(a_{m}-a_{i}\right)}}, \quad i \neq j \neq m .
$$

If $p_{u_{1,2}}$ are the corresponding momenta defined by relations

$$
M_{i}=\frac{2 \varepsilon_{i j m} \gamma_{j} \gamma_{m}\left(a_{j}-a_{m}\right)}{u_{1}-u_{2}}\left(\left(a_{i}-u_{1}\right) p_{u_{1}}-\left(a_{i}-u_{2}\right) p_{u_{2}}\right),
$$

then first integrals $H_{1,2}(2.7)$ satisfy to the following separation relations

$$
4\left(u_{i}-a_{1}\right)\left(u_{i}-a_{2}\right)\left(u_{i}-a_{3}\right) p_{u_{i}}^{2}+G_{i}\left(u_{i}\right)-u_{i} H_{2}+2 H_{1}=0, \quad i=1,2 .
$$

If we take homogeneous polynomials of $N$-th order $G_{1,2}=u^{N}$, the corresponding potentials $V_{1,2}^{(N)}$ satisfy to the well-known recurrence relations

$$
V_{1}^{(1)}=0, \quad V_{2}^{(1)}=1, \quad 2 V_{1}^{(N)}=\rho V_{2}^{(N-1)}, \quad V_{2}^{(N)}=\sigma V_{2}^{(N-1)}-2 V_{1}^{(N-1)},
$$

where

$$
\begin{aligned}
& \sigma=\left(u_{1}+u_{2}\right)=\left(a_{1}+a_{2}+a_{3}\right)-a_{1} \gamma_{1}^{2}-a_{2} \gamma_{2}^{2}-a_{3} \gamma_{3}^{2}, \\
& \rho=u_{1} u_{2}=a_{2} a_{3} \gamma_{1}^{2}+a_{1} a_{3} \gamma_{2}^{2}+a_{1} a_{2} \gamma_{3}^{2} .
\end{aligned}
$$

According to Bogoyavlenskii [12] these potentials are equal to

$$
\begin{aligned}
V_{2}^{(N)} & =\sum_{k=0}^{[N / 2]}(-1)^{k}\left(\begin{array}{c}
N-k \\
k
\end{array}\right) \rho^{k} \sigma^{N-2 k}, \\
2 V_{1}^{(N)} & =\sum_{k=0}^{[(N-1) / 2]}(-1)^{k}\left(\begin{array}{c}
N-k-1 \\
k
\end{array}\right) \rho^{k+1} \sigma^{N-2 k-1} .
\end{aligned}
$$

Here $[z]$ is an integer part of the rational number $z$. If $G_{1,2}(u)=u^{-K}$, one gets rational potentials

$$
V_{2}^{(-K)}=\frac{1 / u_{1}^{K}-1 / u_{2}^{K}}{u_{2}-u_{1}}=\frac{1}{\left(u_{1} u_{2}\right)^{K}} \frac{u_{2}^{K}-u_{1}^{K}}{u_{2}-u_{1}}=-\frac{V_{2}^{(K)}}{\rho^{K}}
$$


and

$$
2 V_{1}^{(-K)}=\frac{u_{2} / u_{1}^{K}-u_{1} / u_{2}^{K}}{u_{2}-u_{1}}=\frac{1}{\left(u_{1} u_{2}\right)^{K}} \frac{u_{2}^{K+1}-u_{1}^{K+1}}{u_{2}-u_{1}}=-\frac{V_{2}^{(K+1)}}{\rho^{K}}
$$

Of course, any linear combination of these polynomial and rational potentials also satisfies (2.9).

For instance, at $N=2$ one gets the Neumann system

$$
\begin{aligned}
& H_{1}^{(2)}=\frac{1}{2}(M, \mathbf{A} M)-a_{2} a_{3} \gamma_{1}^{2}-a_{1} a_{3} \gamma_{2}^{2}-a_{1} a_{2} \gamma_{3}^{2}, \\
& H_{2}^{(2)}=(M, M)+a_{1} \gamma_{1}^{2}+a_{2} \gamma_{2}^{2}+a_{3} \gamma_{3}^{2},
\end{aligned}
$$

and the Braden system at $K=1$

$$
\begin{aligned}
H_{1}^{(-1)} & =\frac{1}{2}(M, \mathbf{A} M)+\frac{a_{1} \gamma_{1}^{2}+a_{2} \gamma_{2}^{2}+a_{3} \gamma_{3}^{2}-\left(\gamma_{1}^{2}+\gamma_{2}^{2}+\gamma_{3}^{2}\right)\left(a_{1}+a_{2}+a_{3}\right)}{a_{2} a_{3} \gamma_{1}^{2}+a_{1} a_{3} \gamma_{2}^{2}+a_{1} a_{2} \gamma_{3}^{2}}, \\
H_{2}^{(-1)} & =(M, M)+\frac{1}{a_{2} a_{3} \gamma_{1}^{2}+a_{1} a_{3} \gamma_{2}^{2}+a_{1} a_{2} \gamma_{3}^{2}} .
\end{aligned}
$$

In [41] Wojciechowski presented another family of potentials associated with other symmetric functions $G_{1,2}(2.11)$ on variables $u_{1,2}$ and parameters $a_{1}, a_{2}, a_{3}$. For instance, if

$$
\begin{aligned}
& F_{1,2}^{(2)}(u)=u\left(u^{2}-\left(a_{1}+a_{2}+a_{3}\right) u+a_{1} a_{2}+a_{1} a_{3}+a_{2} a_{3}\right), \\
& F_{1,2}^{(3)}(u)=u\left(u-a_{1}\right)\left(u-a_{2}\right)\left(u-a_{3}\right)
\end{aligned}
$$

then the second integrals of motion read as

$$
\begin{aligned}
& H_{2}^{(2)}=(M, M)+\sum a_{i}^{2} \gamma_{i}^{2}-\left(\sum a_{i} \gamma_{i}^{2}\right)^{2} \\
& H_{2}^{(3)}=(M, M)+\sum a_{i}^{3} \gamma_{i}^{2}-2\left(\sum a_{i} \gamma_{i}^{2}\right)\left(\sum a_{k}^{2} \gamma_{k}^{2}\right)+\left(\sum a_{i} \gamma_{i}^{2}\right)^{3} .
\end{aligned}
$$

More complicated functions $G_{1,2}(2.11)$ yield more complicated potentials, for example, rational functions

$$
G_{1,2}(u)=\sum_{i=1}^{3} b_{i} b_{j} u+\frac{\left(a_{1}-a_{3}\right)\left(a_{1}-a_{2}\right) b_{1}^{2}}{u-a_{1}}+\frac{\left(a_{2}-a_{3}\right)\left(a_{2}-a_{1}\right) b_{2}^{2}}{u-a_{2}}+\frac{\left(a_{3}-a_{1}\right)\left(a_{3}-a_{2}\right) b_{3}^{2}}{u-a_{3}}
$$

give rise to the Rosochatius potentials [31]

$$
\begin{aligned}
& H_{1}^{(\mathrm{Ros})}=\frac{1}{2}(M, \mathbf{A} M)+\frac{b_{1}^{2}\left(a_{2} \gamma_{3}^{2}+a_{3} \gamma_{2}^{2}\right)}{\gamma_{1}^{2}}+\frac{b_{2}^{2}\left(a_{1} \gamma_{3}^{2}+a_{3} \gamma_{1}^{2}\right)}{\gamma_{2}^{2}}+\frac{b_{3}^{2}\left(a_{1} \gamma_{2}^{2}+a_{2} \gamma_{1}^{2}\right)}{\gamma_{3}^{2}} \\
& H_{2}^{(\mathrm{Ros})}=(M, M)+\frac{b_{1}^{2}}{\gamma_{1}^{2}}+\frac{b_{2}^{2}}{\gamma_{2}^{2}}+\frac{b_{3}^{2}}{\gamma_{3}^{2}}
\end{aligned}
$$

up to the constant terms, see also [24].

In [19] Dragović considered functions $G_{1,2}(2.11)$, which are the Laurent polynomials, and ingeniously coupled the corresponding potentials $V_{1,2}$ together with the Appell hypergeometric function.

These familiar well-studied potentials are permanently rediscovered both in holonomic [24, 39] and nonholonomic mechanics [22, 29]. 


\section{Suslov problem}

One of the most widely known mechanical nonholonomic systems is the Suslov problem describing motion of a rigid body under the following constraint on its angular velocity

$$
(\omega, a)=0,
$$

where $a$ is a fixed unit vector in the body frame [33]. It means that there is no twisting around this vector $a$.

Imposing this constraint we have to add some terms with the Lagrangian multiplier to the initial Hamiltonian vector field

$$
\mathbf{I} \dot{\omega}=\mathbf{I} \times \omega+\gamma \times \frac{\partial V_{1}(\gamma)}{\partial \gamma}+\lambda a, \quad \dot{\gamma}=\gamma \times \omega .
$$

Differentiating the constraint (3.1) by time and using the equation of motion we obtain

$$
\lambda=\frac{1}{\left(\mathbf{I}^{-1} a, a\right)}\left(\mathbf{I}^{-1} a, \mathbf{I} \times \omega+\frac{1}{2} \gamma \times \frac{\partial V_{1}(\gamma)}{\partial \gamma}\right) .
$$

Vector field (3.2) preserves the mechanical energy

$$
H_{1}=(M, \mathbf{A} M)+V_{1}(\gamma)
$$

and the geometric constants of motion

$$
C_{1}=(\gamma, \gamma)=1, \quad C_{2}=(\omega, a)=0,
$$

which allows us to remove the redundant variable from the calculations

$$
\gamma_{3}=\sqrt{1-\gamma_{1}^{2}-\gamma_{2}^{2}}
$$

If we assume that $a$ is an eigenvector of the tensor of inertia [14, 20, 29], i.e., that tensor of inertia is diagonal

$$
\mathbf{I}=\left(\begin{array}{ccc}
I_{1} & 0 & 0 \\
0 & I_{2} & 0 \\
0 & 0 & I_{3}
\end{array}\right),
$$

and vector $a$ is equal to $a=(0,0,1)$ in some coordinate frame, the constraint is trivial

$$
\omega_{3}=0 .
$$

Substituting the standard anzats for the second integral of motion

$$
H_{2}=f_{1}(\gamma) \omega_{1}^{2}+f_{2}(\gamma) \omega_{2}^{2}+f_{3}(\gamma) \omega_{1} \omega_{2}+V_{2}(\gamma)
$$

with unknown functions $f_{k}$ and $V_{2}$ on the components $\gamma_{1,2}$ of the Poisson vector in the equation $\dot{H}_{2}=0$ we obtain the well-known Bertrand-Darboux equation on potential $[9,17]$.

Proposition 2. For vector field (3.2) the following statements are equivalent:

1. There is an additional independent first integral of second order in velocities

$$
\begin{aligned}
H_{2}= & \left(\frac{\alpha \sqrt{I_{1} I_{2}}}{2} \gamma_{1}^{2}+\beta_{1} \sqrt{I_{1}} \gamma_{1}+\frac{\gamma_{11} \sqrt{I_{1}}}{2 \sqrt{I_{2}}}\right) \omega_{1}^{2} \\
& +\left(\frac{\alpha \sqrt{I_{1} I_{2}}}{2} \gamma_{2}^{2}+\beta_{2} \sqrt{I_{2}} \gamma_{2}+\frac{\gamma_{22} \sqrt{I_{2}}}{2 \sqrt{I_{1}}}\right) \omega_{2}^{2} \\
& +\left(\alpha \sqrt{I_{1} I_{2}} \gamma_{1} \gamma_{2}+\beta_{1} \sqrt{I_{1}} \gamma_{2}+\beta_{2} \sqrt{I_{2}} \gamma_{1}+\gamma_{12}\right) \omega_{1} \omega_{2}+V_{2}(\gamma) .
\end{aligned}
$$


2. Potential $V_{1}$ satisfies the Bertrand-Darboux equation (2.2) with

$$
q_{1}=\gamma_{1} \sqrt{I_{2}}, \quad q_{2}=\gamma_{2} \sqrt{I_{1}} \text {. }
$$

3. Potential $V_{1}$ is separable. A characteristic coordinate system for the Bertrand-Darboux equation provides separation for $V_{1}$ and can be taken as one of the following four orthogonal coordinate systems on the $q_{1,2}$-plane: elliptic, parabolic, polar or Cartesian.

The proof is completely similar to the one for the original Bertrand-Darboux theorem [17, 32].

This result allows us to suppose that the nonholonomic Suslov system is equivalent to the holonomic motion on the plane with coordinates $q_{1}, q_{2}$ after some singular change of time, but its study is out of the framework of the present note.

Another reduction to the Bertrand-Darboux equation was proposed in [29]. Integrable vector field remains integrable for any fixed value of mechanical energy $H_{1}$, for instance on the zeroenergy hypersurface

$$
H_{1}=0 .
$$

In the Hamiltonian case the separation of variables of this null Hamilton-Jacobi equation is equivalent to the ordinary separation of the image of the original Hamiltonian under a generalized Jacobi-Maupertuis transformation [8].

If we substitute potential

$$
V_{1}=-\frac{1}{2}\left(\frac{m u_{1}^{2}}{I_{2}}+\frac{m u_{2}^{2}}{I_{1}}\right)
$$

and velocities

$$
\omega_{1}=\frac{m u_{2}}{I_{1}}, \quad \omega_{2}=-\frac{\mu_{1}}{I_{2}}, \quad \omega_{3}=0
$$

from Theorem 1 in [29] into the first integral $H_{1}(3.3)$, one gets $H_{1}=0$. Here $\mu_{1,2}$ are functions on the components of the Poisson vector $\gamma_{1,2}$, which satisfy equations (3.2)

$$
\dot{\gamma_{1}}=-\sqrt{1-\gamma_{1}^{2}-\gamma_{2}^{2}} \frac{\mu_{1}}{I_{2}}, \quad \dot{\gamma_{2}}=-\sqrt{1-\gamma_{1}^{2}-\gamma_{2}^{2}} \frac{\mu_{2}}{I_{1}} .
$$

Differentiating these equations with respect to time we obtain the Newton equations (2.1) on $q_{1,2}$ (3.4) with forces labelled by two functions $\mu_{1,2}\left(q_{1}, q_{2}\right)$. Thus, on the zero-energy hypersurface of the initial Hamiltonian one gets an initial Bertrand problem with the well-known solution. Of course, on this zero-energy hypersurface we can find other solutions associated with nonitegrable potentials on the whole phase space. In [29] such potentials were called quasiimplicitly integrable or locally integrable potentials.

\section{Veselova system}

Let us consider the nonholonomic Veselova system describing the motion of a rigid body under the following constraint

$$
(\omega, \gamma)=0
$$

where $\gamma$ is a unit Poisson vector fixed in space [40]. It means that there is no twisting around vector $\gamma$. 
According to [40] this constraint shifts the initial Hamiltonian vector field (2.4)

$$
\dot{M}=M \times \omega+\lambda \gamma, \quad \dot{\gamma}=\gamma \times \omega,
$$

where the Lagrangian multiplier $\lambda$ is chosen so that the constraint (4.1) is satisfied at any time

$$
\lambda=\frac{(\mathbf{A} M \times M, \mathbf{A} \gamma)}{(\mathbf{A} \gamma, \gamma)} .
$$

There are integrals of motion of second order in momenta

$$
H_{1}=\frac{1}{2}(M, \mathbf{A} M), \quad H_{2}=(M, M)-(\gamma, \gamma)^{-1}(\gamma, M)^{2}
$$

and two geometric constants of motion

$$
C_{1}=(\gamma, \gamma), \quad C_{2}=(\gamma, \omega)=0 .
$$

In the presence of the potential field equations of motion (4.2) become

$$
\dot{M}=M \times \omega+\lambda \gamma+\gamma \times \frac{\partial W_{1}(\gamma)}{\partial \gamma}, \quad \dot{\gamma}=\gamma \times \omega
$$

where

$$
\lambda=\frac{\left(\mathbf{A} M \times M+\gamma \times \partial W_{1}(\gamma) / \partial \gamma, \mathbf{A} \gamma\right)}{(\mathbf{A} \gamma, \gamma)} .
$$

As usual, functions $C_{1,2}$ remain constants of motion, and we can exclude the redundant variable

$$
\gamma_{3}=\sqrt{1-\gamma_{1}^{2}-\gamma_{2}^{2}}
$$

Vector field (4.3) is a conformally Hamiltonian field, see, for instance, [15, 36]. Substituting the following anzats for integrals of motion

$$
H_{1}=\frac{1}{2}(M, \mathbf{A} M)+W_{1}(\gamma), \quad H_{2}=(M, M)-(\gamma, \gamma)^{-1}(\gamma, M)^{2}+W_{2}(\gamma)
$$

in $\dot{H}_{1,2}=0$ one gets two first-order equations on potentials $W_{1,2}$

$$
\begin{aligned}
& 2\left(\gamma_{2}^{2}\left(a_{2}^{-1}-a_{3}^{-1}\right)-a_{2}^{-1}\right) \partial_{1} W_{1}-2 \gamma_{1} \gamma_{2}\left(a_{1}^{-1}-a_{3}^{-1}\right) \partial_{2} W_{1}+\partial_{1} W_{2}=0 \\
& 2\left(\gamma_{1}^{2}\left(a_{1}^{-1}-a_{3}^{-1}\right)-a_{1}^{-1}\right) \partial_{2} W_{1}-2 \gamma_{1} \gamma_{2}\left(a_{2}^{-1}-a_{3}^{-1}\right) \partial_{1} W_{1}+\partial_{2} W_{2}=0 .
\end{aligned}
$$

Proposition 3. After the inversion of parameters $a_{k} \rightarrow a_{k}^{-1}$ and substitution

$$
W_{2}=2 V_{1}, \quad W_{1}=\frac{V_{2}}{2}
$$

equations (4.5) coincide with equations (2.9) for potentials on the two-dimensional sphere.

Thus, all the integrable potentials for the nonholomic Veselova system are easily expressed via well-known integrable potentials $V_{1,2}$ for the holonomic system on the two-dimensional sphere. For functions $G_{1,2}(2.11)$ which are the Laurent polynomials in $u_{1,2}$ these expressions were found in [20]. We only want to note that it is true for any integrable potentials.

Following Theorem 2 in [29] let us fix the values of velocities by equations

$$
I_{1} \omega_{1} \gamma_{2}-I_{2} \omega_{2} \gamma_{1}-\Psi_{2}=0, \quad p \omega_{3}-\Psi_{1}=0, \quad \omega_{1} \gamma_{1}+\omega_{2} \gamma_{2}+\omega_{3} \gamma_{3}=0,
$$


where $p=\sqrt{I_{1} I_{2} I_{3}\left(\frac{\gamma_{1}^{2}}{I_{1}}+\frac{\gamma_{2}^{2}}{I_{2}}+\frac{\gamma_{3}^{2}}{I_{3}}\right)}$ and $\Psi_{1,2}(\gamma)$ are functions on $\gamma$. Substituting these velocities and potential

$$
W_{1}=-\frac{\Psi_{1}^{2}+\Psi_{2}^{2}}{2\left(I_{1} \gamma_{2}^{2}+I_{2} \gamma_{1}^{2}\right)}
$$

into the mechanical energy $H_{1}(4.4)$ one gets $H_{1}=0$.

The remaining three equations of motion of the components of the Poisson vector $\gamma$ are easily reduced to equations of motion for the holonomic particle on the sphere with forces labelled by two functions $\Psi_{1,2}(\gamma)$. Thus, on the zero-energy hypersurface of the initial Hamiltonian one gets standard characteristic equation (2.10) with the well-known solutions.

\section{Chaplygin ball}

As in [16] we consider the rolling of a dynamically balanced ball on a horizontal absolutely rough table without slipping or sliding. 'Dynamically balanced' means that the geometric center coincides with the center of mass, but mass distribution is not assumed to be homogeneous. Because of the roughness of the table this ball cannot slip, but it can turn about the vertical axis without violating the constraints.

After reduction [16] motion of the Chaplygin ball is defined by the following vector field

$$
\dot{M}=M \times \omega, \quad \dot{\gamma}=\gamma \times \omega .
$$

Here $M$ is the angular momentum of the ball with respect to the contact point, $\omega$ is the angular velocity vector of the rolling ball. Its mass, inertia tensor and radius will be denoted by $m$, $\mathbf{I}=\operatorname{diag}\left(I_{1}, I_{2}, I_{3}\right)$ and $b$ respectively. All the vectors are expressed in the so-called body frame, which is firmly attached to the ball, and its axes coincide with the principal inertia axes of the ball.

The angular velocity vector is equal to $\omega=\mathbf{A}_{g} M$, here matrix

$$
\mathbf{A}_{g}=\mathbf{A}+d g(\gamma) \mathbf{A} \gamma \otimes \gamma \mathbf{A}
$$

is defined by the nondegenerate matrix $\mathbf{A}(2.5)$ and function

$$
\mathrm{g}(\gamma)=\frac{1}{1-d\left(a_{1} \gamma_{1}^{2}+a_{2} \gamma_{2}^{2}+a_{3} \gamma_{3}^{2}\right)}, \quad d=m b^{2} .
$$

It is easy to prove that vector field (5.1) preserves two polynomials of second order in momenta

$$
H_{1}=\frac{1}{2}\left(M, \mathbf{A}_{g} M\right), \quad H_{2}=(M, M)
$$

and two geometric constants of motion

$$
C_{1}=\gamma_{1}^{2}+\gamma_{2}^{2}+\gamma_{3}^{2}=1, \quad C_{2}=\gamma_{1} M_{1}+\gamma_{2} M_{2}+\gamma_{3} M_{3},
$$

see details in $[13,34,37]$.

Indeed, equations of motion of the ball in the potential field

$$
\dot{M}=M \times \omega+\gamma \times \frac{\partial U_{1}(\gamma)}{\partial \gamma}, \quad \dot{\gamma}=\gamma \times \omega
$$

have the same form as the equations (2.6) in rigid body dynamics. In fact, the principal difference between holonomic and nonholonomic systems is hidden within the relation of the angular velocity to the angular momentum. 
According to [35, 36], integrals of motion for the Veselova system are expressed via the integrals of motion for the Chaplygin ball. So, we can easily express integrable potentials for the Chaplygin system via integrable potentials for the Veselova system $W_{1,2}$ (4.5) and then via integrable potentials $V_{1,2}$ (2.9) for the holonomic system on the two-dimensional sphere. Of course, we can get the same result by directly substituting standard ansatz

$$
H_{1}=\frac{1}{2}\left(M, \mathbf{A}_{g} M\right)+U_{1}(\gamma), \quad H_{2}=(M, M)+U_{2}(\gamma)
$$

in $\dot{H}_{1,2}=0$ we obtain

$$
\begin{aligned}
& a_{1}\left(a_{2}-a_{3}\right) \gamma_{1} \gamma_{2} \partial_{1} U_{2}+a_{1}\left(\left(a_{2}-a_{3}\right) \gamma_{2}^{2}+a_{3}-1\right) \partial_{2} U_{2}+2 \mathrm{~g}^{-1} \partial_{2} U_{1}=0, \\
& a_{2}\left(\left(a_{1}-a_{3}\right) \gamma_{1}^{2}+a_{3}-1\right) \partial_{1} U_{2}+a_{2}\left(a_{1}-a_{3}\right) \gamma_{1} \gamma_{2} \partial_{2} U_{2}-2 \mathrm{~g}^{-1} \partial_{1} U_{1}=0, \\
& a_{3} \gamma_{2}\left(\left(a_{1}-a_{2}\right) \gamma_{1}+a_{2}-1\right) \partial_{1} U_{2}+a_{3} \gamma_{1}\left(\left(a_{1}-a 2\right) \gamma_{2}^{2}-a_{1}+1\right) \partial_{2} U_{2} \\
& \quad \quad+2 \mathrm{~g}^{-1}\left(\gamma_{1} \partial_{2} U_{1}-\gamma_{2} \partial_{1} U_{1}\right)=0 .
\end{aligned}
$$

This system of equations has only one solution

$$
U_{1}=-\frac{1}{2}\left(a_{2} a_{3} \gamma_{1}^{2}+a_{1} a_{3} \gamma_{2}^{2}+a_{1} a_{2} \gamma_{3}^{2}\right), \quad U_{2}=a_{1} \gamma_{1}^{2}+a_{2} \gamma_{2}^{2}+a_{3} \gamma_{3}^{2},
$$

which coincides with the single solution of the initial system (2.8) associated with the Clebsch model. This integrable potential has been found in [27].

At $C_{2}=0$ conditions $\dot{H}_{1,2}=0$ are thus

$$
\begin{aligned}
& \mathrm{g} \gamma_{1} \gamma_{2}\left(a_{2}\left(a_{1}-a_{3}\right) \gamma_{1}^{2}+a_{1}\left(a_{2}-a_{3}\right) \gamma_{2}^{2}-a_{1} a_{2}+\left(a_{1}+a_{2}-1\right) a_{3}\right) \partial_{1} U_{2} \\
& \quad-\mathrm{g}\left(a_{1}\left(x_{1}^{2}+x_{2}^{2}-1\right)\left(a_{2} x_{2}^{2}-1\right)-a_{3}\left(a_{1}\left(x_{2}^{4}-1\right)+x_{1}^{2}\left(a_{2} x_{2}^{2}-1\right)\right)\right) \partial_{2} U_{2} \\
& \quad-2 \gamma_{1} \gamma_{2} \partial_{1} U_{1}-2\left(\gamma_{2}^{2}-1\right) \partial_{2} U_{1}=0, \\
& \mathrm{~g}\left(a_{2}\left(x_{1}^{2}+x_{2}^{2}-1\right)\left(a_{1} x_{1}^{2}-1\right)-a_{3}\left(a_{2}\left(x_{1}^{4}-1\right)+x_{2}^{2}\left(a_{1} x_{1}^{2}-1\right)\right)\right) \partial_{1} U_{2} \\
& \quad-\mathrm{g} \gamma_{1} \gamma_{2}\left(a_{2}\left(a_{1}-a_{3}\right) \gamma_{1}^{2}+a_{1}\left(a_{2}-a_{3}\right) \gamma_{2}^{2}-a_{1} a_{2}+\left(a_{1}+a_{2}-1\right) a_{3}\right) \partial_{2} U_{2} \\
& \quad+2\left(x_{1}^{2}-1\right) \partial_{1} U_{1}+2 \gamma_{1} \gamma_{2} \partial_{2} U_{1}=0 .
\end{aligned}
$$

Here $g \equiv g(\gamma)$ is the function defined by (5.2). If we change the parameters

$$
e_{1}=\frac{a_{1}}{1-a_{1}}, \quad e_{2}=\frac{a_{2}}{1-a_{2}}, \quad e_{3}=\frac{a_{3}}{1-a_{3}}
$$

and substitute in (5.5)

$$
\begin{aligned}
& 2 U_{1}=\left(e_{2} e_{3} \gamma_{1}^{2}+e_{1} e_{3} \gamma_{2}^{2}+e_{1} e_{2} \gamma_{3}^{2}\right) V_{2}+2 V_{1}, \\
& U_{2}=d\left(1+\left(e_{1}+e_{2}+e_{3}\right)-e_{1} \gamma_{1}^{2}-e_{2} \gamma_{2}^{2}-e_{3} \gamma_{3}^{2}+e_{2} e_{3} \gamma_{1}^{2}+e_{1} e_{3} \gamma_{2}^{2}+e_{1} e_{2} \gamma_{3}^{2}\right) V_{2},
\end{aligned}
$$

then the equations (5.5) become

$$
\begin{aligned}
& \left(\gamma_{2}^{2}\left(e_{2}-e_{3}\right)-e_{2}\right) \partial_{1} V_{1}-\gamma_{1} \gamma_{2}\left(e_{1}-e_{3}\right) \partial_{2} V_{2}+2 \partial_{1} V_{1}=0 \\
& \left(\gamma_{1}^{2}\left(e_{1}-e_{3}\right)-e_{1}\right) \partial_{2} V_{1}-\gamma_{1} \gamma_{2}\left(e_{2}-e_{3}\right) \partial_{1} V_{2}+2 \partial_{2} V_{1}=0 .
\end{aligned}
$$

It is easy to see that this system coincides with the initial system of equations (2.9) defining integrable potentials on the sphere up to $a_{k} \rightarrow e_{k}$.

Thus, for the Chaplygin ball imposition of the nonholonomic constraint leads to deformation of potentials (5.6) and to replacement of parameters $a_{k} \rightarrow e_{k}$. 
Proposition 4. At $C_{2}=0$ conformally Hamiltonian vector field (5.3) has two integrals of motion (5.4) with potentials

$$
2 U_{1}=\rho V_{2}+2 V_{1}, \quad U_{2}=d(\rho+\sigma+1) V_{2} .
$$

Here $V_{1,2}$ are integrable potentials on the sphere (2.11) after replacement of parameters $a_{k} \rightarrow e_{k}$, and

$$
\begin{aligned}
& \sigma=\left(\gamma_{1}^{2}+\gamma_{2}^{2}+\gamma_{3}^{2}\right)\left(e_{1}+e_{2}+e_{3}\right)-e_{1} \gamma_{1}^{2}+e_{2} \gamma_{2}^{2}+e_{3} \gamma_{3}^{2}, \\
& \rho=e_{2} e_{3} \gamma_{1}^{2}+e_{1} e_{3} \gamma_{2}^{2}+e_{1} e_{2} \gamma_{3}^{2}
\end{aligned}
$$

are the same polynomials of second order in variables $\gamma$ as above (2.13).

Following to S.A. Chaplygin [16] we can introduce the sphero-conical coordinates $u_{1}, u_{2}$

$$
\gamma_{i}=\sqrt{\frac{\left(u_{1}-e_{i}\right)\left(u_{2}-e_{i}\right)}{\left(e_{j}-e_{i}\right)\left(e_{m}-e_{i}\right)}}, \quad i \neq j \neq m
$$

and explicitly present some solutions of the Bertrand-Darboux type equations (5.5). Namely, integrals of motion $H_{1,2}$ (5.4) satisfy the separation relations

$$
\frac{4\left(e_{1}-u_{i}\right)\left(e_{2}-u_{i}\right)\left(e_{3}-u_{i}\right)}{d\left(e_{1}+1\right)\left(e_{2}+1\right)\left(e_{3}+1\right)} p_{u_{i}}^{2}+G_{i}\left(u_{i}\right)-\frac{u_{i} H_{2}}{d\left(u_{i}+1\right)}+2 H_{1}=0, \quad i=1,2,
$$

which can be considered as gentle deformation of the initial relations (2.12). Thus, separable potentials in this case read as

$$
\begin{aligned}
U_{2} & =\frac{d\left(u_{1}+1\right)\left(u_{2}+1\right)\left(G_{1}\left(u_{1}\right)-G_{2}\left(u_{2}\right)\right)}{u_{2}-u_{1}}, \\
U_{1} & =\frac{u_{1} u_{2}\left(G_{1}\left(u_{1}\right)-G_{2}\left(u_{2}\right)\right)+u_{2} G_{1}\left(u_{1}\right)-u_{1} G_{2}\left(u_{2}\right)}{2\left(u_{2}-u_{1}\right)} .
\end{aligned}
$$

The passage to limit $d \rightarrow 0$ reduces equations of motion for the Chaplygin ball (5.3) to the standard Euler-Poisson equations. However, at $d \rightarrow 0$ we have to simultaneously change the definition of the second potential $U_{2}$ in (5.6) and, therefore, we present another family of solutions for equations (5.5).

Let us introduce variables $v_{1,2}$

$$
\gamma_{i}=\sqrt{\frac{\left(1-d a_{j}\right)\left(1-d a_{m}\right)}{\left(1-d v_{1}\right)\left(1-d v_{2}\right)}} \cdot \sqrt{\frac{\left(v_{1}-a_{i}\right)\left(v_{2}-a_{i}\right)}{\left(a_{j}-a_{i}\right)\left(a_{m}-a_{i}\right)}}, \quad i \neq j \neq m,
$$

and the conjugated momenta $p_{v_{1,2}}$, see [34] for details. In this variables the separated relations have the following form

$$
4\left(1-d v_{i}\right)\left(v_{i}-a_{1}\right)\left(v_{i}-a_{2}\right)\left(v_{i}-a_{3}\right) p_{v_{i}}^{2}+U_{i}\left(v_{i}\right)+v_{i} H_{2}-2 H_{1}=0, \quad i=1,2,
$$

and integrable potentials

$$
U_{2}=\frac{G_{1}\left(v_{1}\right)-G_{2}\left(v_{2}\right)}{v_{2}-v_{1}}, \quad U_{1}=\frac{v_{2} G_{1}\left(v_{1}\right)-v_{1} G_{2}\left(v_{2}\right)}{2\left(v_{2}-v_{1}\right)}
$$

are the same functions on variables $v_{1,2}$ as the integrable potentials on the sphere $(2.11)$. 
Proposition 5. At $C_{2}=0$ vector field (5.3) has integrals of motion of second order in velocities (5.4) if potentials $U_{1,2}$ have the same form as integrable potentials on the sphere (2.14)

$$
\begin{aligned}
U_{2}^{(N)} & =\sum_{k=0}^{[N / 2]}(-1)^{k}\left(\begin{array}{c}
N-k \\
k
\end{array}\right) \varrho^{k} \varsigma^{N-2 k}, \\
2 U_{1}^{(N)} & =\sum_{k=0}^{[(N-1) / 2]}(-1)^{k}\left(\begin{array}{c}
N-k-1 \\
k
\end{array}\right) \varrho^{k+1} \varsigma^{N-2 k-1},
\end{aligned}
$$

and

$$
U_{2}^{(-K)}=-\frac{U_{1}^{(K)}}{\varrho^{K}}, \quad 2 U_{1}^{(-K)}=-\frac{U_{1}^{(K+1)}}{\varrho^{K}} .
$$

Of course, any linear combination of these polynomial and rational potentials also satisfies the equations (5.5). These potentials differ from (2.14) by replacement of polynomials $\sigma$ and $\rho$ for the following functions

$$
\begin{aligned}
& \varsigma=g(\gamma)\left(\sigma+d\left(a_{1}\left(a_{2}+a_{3}\right) \gamma_{1}^{2}+a_{2}\left(a_{1}+a_{3}\right) \gamma_{2}^{2}+a_{3}\left(a_{1}+a_{2}\right) \gamma_{3}^{2}\right)\right), \\
& \varrho=g(\gamma)\left(\rho+d a_{1} a_{2} a_{3}\left(\gamma_{1}^{2}+\gamma_{2}^{2}+\gamma_{3}^{2}\right)\right),
\end{aligned}
$$

which at $d=0$ become initial polynomials (2.13).

For instance, at $N=2$ we have the following analogue of the Neumann system

$$
H_{1}^{(2)}=\frac{1}{2}(M, \mathbf{A} M)+\varrho, \quad H_{2}^{(2)}=(M, M)+\varsigma,
$$

and at $K=1$ the following counterpart of the Braden system

$$
H_{1}^{(-1)}=\frac{1}{2}(M, \mathbf{A} M)-\frac{\varsigma}{\varrho}, \quad H_{2}^{(-1)}=(M, M)-\frac{1}{\varrho} .
$$

Of course, we can also single out other families of solutions of the equations (5.5), for instance, see [20].

\section{Nonholonomic oscillator and Heisenberg system}

Let us consider the Lagrangian of the particle in Euclidean space $\mathbb{R}^{3}$

$$
L=\frac{m}{2}\left(\dot{x}^{2}+\dot{y}^{2}+\dot{z}^{2}\right)-V(x, y, z),
$$

where $m$ is the mass of the particle. When this system is subject to a nonholonomic constraint, the resulting mechanical system may or may not preserve energy and the phase space volume, and their integrability and reduction theories are completely different from the Hamiltonian case $[1,18,23]$. In this Section we consider two first-order nonholonomic constraints, which displays all the basic properties of first-order nonholonomic systems in the control theory [11].

The first nonholomic constraint and potential in (6.1) for the so-called nonholonomic oscillator have the following form

$$
f=\dot{z}-k y \dot{x}=0, \quad V=\frac{y^{2}}{2} \quad k \in \mathbb{R},
$$


whereas second constraint and potential in (6.1) for the so-called Heisenberg system read as

$$
f=\dot{z}-(y \dot{x}-x \dot{y})=0, \quad V=0 .
$$

The Heisenberg system (nonholonomic integrator) can be pointed out as a benchmark example of nonholonomic system with a first-order nonintegrable constraint, which mimics the kinematic model of a wheeled mobile robot of the unicycle type.

In generic case at $V(x, y, z)=V(x, y)$ in (6.1) the third degree of freedom also decouples from the rest of the system and after nonholonomic reduction we obtain a two-degrees of freedom system of the Chaplygin type $[11,23,30]$. For the such generalized nonholonomic oscillator the reduced equations of motion are

$$
\dot{x}=\frac{p_{x}}{m}, \quad \dot{y}=\frac{p_{y}}{m}, \quad \dot{p}_{x}=-\frac{1}{1+k^{2} y^{2}}\left(k^{2} y p_{x} p_{y}+\partial_{x} V\right), \quad \dot{p}_{y}=-\partial_{y} V .
$$

For the generalized Heisenberg system the reduced equations of motion read as

$$
\begin{aligned}
& \dot{x}=\frac{p_{x}}{m}, \quad \dot{y}=\frac{p_{y}}{m}, \\
& \dot{p}_{x}=-\frac{\left(x^{2}+1\right) \partial_{x} V+x y \partial_{y} V}{m\left(1+x^{2}+y^{2}\right)}, \quad \dot{p}_{y}=-\frac{\left(y^{2}+1\right) \partial_{y} V+x y \partial_{x} V}{m\left(1+x^{2}+y^{2}\right)} .
\end{aligned}
$$

Below we will study potentials $V(x, y)$ in (6.2) and (6.3), so that the corresponding fourdimensional vector fields $X$ have an additional first integral and possess an invariant volume form. Of course, equations on these potentials have the form of the characteristic equation (2.3) and can be considered as an analogue of the Bertrand-Darboux equation (2.2).

\subsection{The generalised nonholonomic oscillator}

The vector field for the reduced nonholonomic oscillator (6.2) after the following change of variables

$$
p_{1}=\sqrt{k^{2} y^{2}+1} p_{x}, \quad p_{2}=\frac{p_{y}}{\sqrt{k^{2} y^{2}+1}}, \quad q_{1}=x, \quad q_{2}=y
$$

becomes the conformally Hamiltonian vector field

$$
X=-\mu P \mathrm{~d} H_{1}, \quad P=\left(\begin{array}{cc}
0 & \mathrm{I} \\
-\mathrm{I} & 0
\end{array}\right)
$$

with respect to the canonical Poisson bivector $P$ and reduced Hamiltonian

$$
H_{1}=\sum_{i, j=1}^{2} \mathrm{~g}_{i j} p_{i} p_{j}+V\left(q_{1}, q_{2}\right)=\frac{p_{1}^{2}}{2 m}+\frac{p_{2}^{2}\left(k^{2} q_{2}^{2}+1\right)}{2 m}+V\left(q_{1}, q_{2}\right) .
$$

Conformal factor

$$
\mu=\frac{1}{\sqrt{k^{2} q_{2}^{2}+1}}
$$

is a nowhere vanishing smooth function on an open dense subset of the plane $q_{2} \neq \infty$, which defines an invariant volume form $\hat{\Omega}=\mu d q \wedge d p$.

Substituting a linear function in velocities

$$
H_{2}=g_{1}\left(q_{1}, q_{2}\right) p_{1}+g_{2}\left(q_{1}, q_{2}\right) p_{2}
$$


into the equation $\dot{H}_{2}=0$ one gets

$$
g_{1}=c_{1}+c_{2} \ln \left(k q_{2}+\sqrt{k^{2} q_{2}^{2}+1}\right), \quad g_{2}=-\sqrt{k^{2} q_{2}^{2}+1}\left(c_{2} k q_{1}-c_{3}\right)
$$

and

$$
V\left(q_{1}, q_{2}\right)=G\left(-\frac{c_{1} \ln \left(k q_{2}+\sqrt{k^{2} q_{2}^{2}+1}\right)}{k}-c_{2}\left(\frac{k q_{1}^{2}}{2}+\int \frac{\ln \left(k q_{2}+\sqrt{k^{2} q_{2}^{2}+1}\right)}{\sqrt{k^{2} q_{2}^{2}+1}}\right)-c_{3} q_{1}\right) .
$$

If we want to consider a single valued integral $H_{2}$, we have to put $c_{2}=0$ and

$$
c_{3}=0, \quad V=G\left(q_{1}\right) \quad \text { or } \quad c_{1}=0, \quad V=G\left(q_{2}\right) .
$$

Substituting polynomials of second order in velocities

$$
H_{2}=\sum_{i, j=1}^{2} K_{i j}\left(q_{1}, q_{2}\right) p_{i} p_{j}+U\left(q_{1}, q_{2}\right),
$$

where $K_{i j}$ and $U$ are single valued functions on $q_{1}, q_{2}$ in the equation $\dot{H}_{2}=0$, we obtain the following expression for the second integral of motion

$$
H_{2}=\frac{c_{1}\left(k^{2} q_{2}^{2}+k q_{2} \sqrt{k^{2} q_{2}^{2}+1}+1\right)}{k q_{2}+\sqrt{k^{2} q_{2}^{2}+1}} p_{1} p_{2}+c_{2}\left(k^{2} q_{2}^{2}+1\right) p_{2}^{2}+U\left(q_{1}, q_{2}\right),
$$

and the following counterpart of the Bertrand-Darboux equation

$$
\frac{c_{1}}{\sqrt{k^{2} q_{2}^{2}+1}}\left(\left(k^{2} q_{2}^{2}+1\right) \partial_{22} V+k^{2} q_{2} \partial_{2} V-\partial_{11} V\right)-2 c_{2} \partial_{12} V=0 .
$$

This equation has one physical and one formal solution

$$
c_{1}=0, \quad V=G_{1}\left(q_{1}\right)+G_{2}\left(q_{2}\right) \quad \text { and } \quad c_{2}=0, \quad V=G_{1}\left(q_{+}\right)+G_{2}\left(q_{-}\right),
$$

where

$$
q_{ \pm}=q_{1} \pm \frac{\ln \left(k q_{2}+\sqrt{k^{2} q_{2}^{2}+1}\right)}{k} .
$$

Thus, for the nonholonomic oscillator we obtain only trivial perturbations in the framework of the Bertrand-Darboux method.

\subsection{The generalized Heisenberg system}

The vector field for the reduced Heisenberg system (6.3) after the following change of variables

$$
p_{1}=\frac{m\left(\left(1+y^{2}\right) p_{x}-x y p_{y}\right)}{1+x^{2}+y^{2}}, \quad p_{2}=\frac{m\left(\left(1+x^{2}\right) p_{y}-x y p_{x}\right)}{1+x^{2}+y^{2}}, \quad q_{1}=x, \quad q_{2}=y
$$

is conformally Hamiltonian vector field

$$
X=-\mu P \mathrm{~d} H_{1}, \quad P=\left(\begin{array}{cc}
0 & \mathrm{I} \\
-\mathrm{I} & 0
\end{array}\right)
$$

with respect to canonical Poisson bivector $P$ and reduced Hamiltonian

$$
H_{1}=\sum_{i, j=1}^{2} \mathrm{~g}_{i j} p_{i} p_{j}+V\left(q_{1}, q_{2}\right)=\frac{q_{1}^{2}+q_{2}^{2}+1}{2 m}\left(p_{1}^{2}+p_{2}^{2}+\left(q_{1} p_{1}+q_{2} p_{2}\right)^{2}\right)+V\left(q_{1}, q_{2}\right) .
$$


Conformal factor

$$
\mu=\left(1+q_{1}^{2}+q_{2}^{2}\right)^{-1}
$$

is a nowhere vanishing smooth function on an open dense subset of the plane $q_{1,2} \neq \infty$, which defines an invariant volume form $\hat{\Omega}=\mu d q \wedge d p$.

Substituting linear function in velocities

$$
H_{2}=g_{1}\left(q_{1}, q_{2}\right) p_{1}+g_{2}\left(q_{1}, q_{2}\right) p_{2}
$$

into the equation $\dot{H}_{2}=0$ one gets the following first integral

$$
H_{2}=\left(p_{1} q_{1}^{2}+p_{2} q_{1} q_{2}+p_{1}\right) c_{1}+\left(p_{1} q_{1} q_{2}+p_{2} q_{2}^{2}+p_{2}\right) c_{2}+\left(p_{1} q_{2}-p_{2} q_{1}\right) c_{3}, \quad c_{k} \in \mathbb{R},
$$

and potential

$$
V=G\left(\frac{\left(c_{1} q_{1}+c_{2} q_{2}\right)^{2}+2 c_{3}\left(c_{1} q_{2}-c_{2} q_{1}\right)+c_{1}^{2}+c_{2}^{2}-c_{3}^{2}}{\left(c_{1} q_{2}-c_{2} q_{1}-c_{3}\right)^{2}}\right)
$$

depending on the arbitrary function $G$.

Substituting polynomials of second order in velocities

$$
H_{2}=\sum_{i, j=1}^{2} K_{i j}\left(q_{1}, q_{2}\right) p_{i} p_{j}+U\left(q_{1}, q_{2}\right)
$$

into the equation $\dot{H}_{2}=0$ one gets the following expression for the second integral of motion

$$
\begin{aligned}
H_{2} & =\left(p_{1} q_{2}-p_{2} q_{1}\right)\left(p_{1} q_{1}^{2}+p_{2} q_{1} q_{2}+p_{1}\right) c_{1}+\left(p_{1} q_{1}^{2}+p_{2} q_{1} q_{2}+p_{1}\right)\left(p_{1} q_{1} q_{2}+p_{2} q_{2}^{2}+p_{2}\right) c_{2} \\
& +\left(p_{1} q_{2}-p_{2} q_{1}\right)\left(p_{1} q_{1} q_{2}+p_{2} q_{2}^{2}+p_{2}\right) c_{3}-\left(p_{1} q_{2}-p_{2} q_{1}\right)^{2} c_{4} \\
& +\left(q_{2}^{2}\left(q_{1}^{2}+1\right) p_{1}^{2}+2 q_{2}^{3} q_{1} p_{1} p_{2}+\left(q_{2}^{4}+q_{1}^{2}+2 q_{2}^{2}+1\right) p_{2}^{2}\right) c_{5} \\
& +\left(\left(q_{1}^{4}+2 q_{1}^{2}+q_{2}^{2}+1\right) p_{1}^{2}+2 q_{1}^{3} q_{2} p_{1} p_{2}+q_{1}^{2}\left(q_{2}^{2}+1\right) p_{2}^{2}\right) c_{6}+U\left(q_{1}, q_{2}\right) .
\end{aligned}
$$

In this case equation (2.3) looks like

$$
A \partial_{11} V+2 B \partial_{12} V+C \partial_{22} V+\frac{1}{1+q_{1}^{2}+q_{2}^{2}}\left(a \partial_{1} V+b \partial_{2} V\right)=0
$$

where $A, B, C$ are the polynomials of second order in $q_{1,2}$

$$
\begin{aligned}
& A=\left(q_{1}^{2}+1\right)\left(q_{1} c_{1}-c_{2}\right)+q_{2}\left(q_{1}^{2}-1\right) c_{3}+2 q_{1} q_{2}\left(c_{6}-c_{4}\right), \\
& B=q_{2}\left(q_{1}^{2}+1\right) c_{1}+q_{1}\left(q_{2}^{2}+1\right) c_{3}+\left(q_{1}^{2}-q_{2}^{2}\right) c_{4}-\left(q_{1}^{2}+1\right) c_{5}+\left(q_{2}^{2}+1\right) c_{6}, \\
& C=\left(q_{2}^{2}+1\right)\left(q_{2} c_{3}+c_{2}\right)+q_{1}\left(q_{2}^{2}-1\right) c_{1}+2 q_{1} q_{2}\left(c_{4}-c_{5}\right),
\end{aligned}
$$

and $a, b$ are the polynomials of fourth order

$$
\begin{aligned}
a= & \left(2 q_{1}^{4}+2 q_{1}^{2} q_{2}^{2}+5 q_{1}^{2}-q_{2}^{2}+3\right) c_{1}+q_{1}\left(q_{1}^{2}-3 q_{2}^{2}+1\right) c_{2}+2 q_{1} q_{2}\left(q_{1}^{2}+q_{2}^{2}+3\right) c_{3} \\
& -2 q_{2}\left(q_{1}^{2}+q_{2}^{2}+3\right) c_{4}+4 q_{2}\left(q_{1}^{2}+1\right) c_{5}-2 q_{2}\left(q_{1}^{2}-q_{2}^{2}-1\right) c_{6}, \\
b= & 2 q_{1} q_{2}\left(q_{1}^{2}+q_{2}^{2}+3\right) c_{1}+q_{2}\left(3 q_{1}^{2}-q_{2}^{2}-1\right) c_{2}+\left(2 q_{1}^{2} q_{2}^{2}+2 q_{2}^{4}-q_{1}^{2}+5 q_{2}^{2}+3\right) c_{3} \\
& +2 q_{1}\left(q_{1}^{2}+q_{2}^{2}+3\right) c_{4}-2 q_{1}\left(q_{1}^{2}-q_{2}^{2}+1\right) c_{5}-4 q_{1}\left(q_{2}^{2}+1\right) c_{6} .
\end{aligned}
$$

Following Darboux [17] we can find the canonical form of the corresponding Killing tensor and a few families of solutions to this equation. For instance, if $c_{6}=1$ and other constants of 
integration are equal to zero, then solutions of the equation $\dot{H}_{2}=0$ are labelled by two arbitrary functions $G_{1,2}$

$$
\begin{aligned}
& V\left(q_{1}, q_{2}\right)=\frac{q_{1}^{2}+q_{2}^{2}+1}{2 m} G_{1}\left(q_{2}\right)+\frac{1}{2 m} G_{2}\left(\frac{q_{2}^{2}+1}{q_{1}^{2}}\right), \\
& U\left(q_{1}, q_{2}\right)=q_{1}^{2} G_{1}\left(q_{2}\right)+G_{2}\left(\frac{q_{2}^{2}+1}{q_{1}^{2}}\right) .
\end{aligned}
$$

If $c_{4}=1$ and other constants of integration are equal to zero, then we have solution

$$
V\left(q_{1}, q_{2}\right)=G_{1}(r)-\frac{r^{2}+1}{2 m r^{2}} G_{2}(\varphi), \quad U\left(q_{1}, q_{2}\right)=G_{2}(\varphi)
$$

associated with polar coordinates on the plane

$$
q_{1}=r \cos \varphi, \quad q_{2}=r \sin \varphi .
$$

Here $G_{1,2}$ are arbitrary functions. In similar manner we can get solutions associated with parabolic and elliptic coordinates on the plane, but they are bulky and, therefore, we do not present these solution explicitly.

In order to get these solutions we can also use the Birkhoff method. Namely, let us consider a general natural system of two degrees of freedom described in certain generalized coordinates by the following Lagrangian

$$
L=\sum_{i, j=1}^{2} \mathrm{~g}_{i j}(q) \frac{d q_{i}}{d t} \frac{d q_{j}}{d t}-V\left(q_{1}, q_{2}\right) .
$$

According to Birkhoff [10] using change of time $t \rightarrow \tau$ and coordinates $\left(q_{1}, q_{2}\right) \rightarrow(x, y)$ this Lagrangian can always be reduced to the form of

$$
L=\left(\frac{d x}{d \tau}\right)^{2}+\left(\frac{d y}{d \tau}\right)^{2}-U(x, y) .
$$

Thus, taking the well-known solutions $U(x, y)$ of the classical Bertrand-Darboux equation (2.2) and applying the inverse Birkhoff transformation we are able to obtain integrable potentials $V\left(q_{1}, q_{2}\right)$ for the two-dimensional holonomic system with nonstandard metric.

We can apply this method for the given nonholonomic case because the corresponding vector field $X(6.5)$ is a conformally Hamiltonian vector field [30,36], i.e., it can be reduced to a Hamiltonian vector field by changing of time. Recall, that equation $\dot{H}_{2}=0$ (1.4) is invariant with respect to change of time, which we have to use both in the Birkhoff method and in the reduction of the conformally Hamiltonian vector field to the Hamiltonian one.

\section{Conclusion}

In this paper, we consider perturbations of the five well-known two-dimensional nonholonomic systems, which are integrable by the Euler-Jacobi theorem. We show that the BertrandDarboux method is applicable to these systems and that all the obtained Bertrand-Darboux type equations in nonholonomic case can be reduced to the Bertrand-Darboux type equations in holonomic case. Consequently, we can directly obtain all the possible integrable potentials for these nonholonomic systems directly from the well-known integrable potentials of the Hamiltonian mechanics. 


\section{Acknowledgements}

We are greatly indebted B. Jovanović and the anonymous referees for a relevant contribution to improve the paper. The work on the revised, final version of this paper was supported by Russian Science Foundation (project No 15-12-20035).

\section{References}

[1] Bates L., Cushman R., What is a completely integrable nonholonomic dynamical system?, Rep. Math. Phys. 44 (1999), 29-35.

[2] Benenti S., Orthogonal separable dynamical systems, in Differential Geometry and its Applications (Opava, 1992), Math. Publ., Vol. 1, Editors O. Kowalsky, D. Krupka, Silesian University Opava, Opava, 1993, 163184.

[3] Benenti S., Geometrical aspects of the dynamics of non-holonomic systems, Rend. Sem. Mat. Univ. Politec. Torino 54 (1996), 203-212.

[4] Benenti S., Intrinsic characterization of the variable separation in the Hamilton-Jacobi equation, J. Math. Phys. 38 (1997), 6578-6602.

[5] Benenti S., A 'user-friendly' approach to the dynamical equations of non-holonomic systems, SIGMA $\mathbf{3}$ (2007), 036, 33 pages, math.DS/0703043.

[6] Benenti S., A general method for writing the dynamical equations of nonholonomic systems with ideal constraints, Regul. Chaotic Dyn. 13 (2008), 283-315.

[7] Benenti S., The non-holonomic double pendulum: an example of non-linear non-holonomic system, Regul. Chaotic Dyn. 16 (2011), 417-442.

[8] Benenti S., Chanu C., Rastelli G., Variable-separation theory for the null Hamilton-Jacobi equation, J. Math. Phys. 46 (2005), 042901, 29 pages.

[9] Bertrand J.M., Mémoire sur quelques-unes des forms les plus simples que puissent présenter les intégrales des équations différentielles du mouvement d'un point matériel, J. Math. Pures Appl. 2 (1857), 113-140.

[10] Birkhoff G.D., Dynamical systems, American Mathematical Society Colloquium Publications, Vol. 9, Amer. Math. Soc., Providence, R.I., 1966.

[11] Bloch A.M., Nonholonomic mechanics and control, Interdisciplinary Applied Mathematics, Vol. 24, SpringerVerlag, New York, 2003.

[12] Bogoyavlenskiŭ O.I., Integrable cases of rigid-body dynamics and integrable systems on spheres $S^{n}, I z v$. Akad. Nauk SSSR Ser. Mat. 49 (1985), 899-915.

[13] Borisov A.V., Kilin A.A., Mamaev I.S., The problem of drift and recurrence for the rolling Chaplygin ball, Regul. Chaotic Dyn. 18 (2013), 832-859.

[14] Borisov A.V., Mamaev I.S., Bizyaev I.A., The hierarchy of dynamics of a rigid body rolling without slipping and spinning on a plane and a sphere, Regul. Chaotic Dyn. 18 (2013), 277-328.

[15] Borisov A.V., Mamaev I.S., Tsiganov A.V., Non-holonomic dynamics and Poisson geometry, Russ. Math. Surv. 69 (2014), 481-538.

[16] Chaplygin S.A., On a ball's rolling on a horizontal plane, Regul. Chaotic Dyn. 7 (2002), 131-148.

[17] Darboux G., Sur un probléme de mécanique, Arch. Néerl. 6 (1901), 371-376.

[18] de M. Rios P., Koiller J., Non-holonomic systems with symmetry allowing a conformally symplectic reduction, in New Advances in Celestial Mechanics and Hamiltonian Systems, Kluwer/Plenum, New York, 2004, 239-252, math-ph/0203013.

[19] Dragović V., The Appell hypergeometric functions and classical separable mechanical systems, J. Phys. A: Math. Gen. 35 (2002), 2213-2221, math-ph/0008009.

[20] Dragović V., Gajić B., Jovanović B., Generalizations of classical integrable nonholonomic rigid body systems, J. Phys. A: Math. Gen. 31 (1998), 9861-9869.

[21] Eisenhart L.P., Separable systems of Stackel, Ann. of Math. 35 (1934), 284-305.

[22] Fedorov Y.N., Jovanović B., Nonholonomic LR systems as generalized Chaplygin systems with an invariant measure and flows on homogeneous spaces, J. Nonlinear Sci. 14 (2004), 341-381, math-ph/0307016. 
[23] Guha P., The role of the Jacobi last multiplier in nonholonomic systems and almost symplectic structure, Preprint, IHES/M/13/17, 2013.

[24] Jovanović B., Integrable perturbations of billiards on constant curvature surfaces, Phys. Lett. A 231 (1997), $353-358$.

[25] Kalnins E.G., Miller Jr. W., Separation of variables on $n$-dimensional Riemannian manifolds. I. The $n$ sphere $S^{n}$ and Euclidean $n$-space $\mathbf{R}^{n}$, J. Math. Phys. 27 (1986), 1721-1736.

[26] Kozlov V.V., Methods of qualitative analysis in the dynamics of a rigid body, Moscow State University, Moscow, 1980.

[27] Kozlov V.V., On the integration theory of equations of nonholonomic mechanics, Regul. Chaotic Dyn. 7 (2002), 161-176, nlin.SI/0503027.

[28] Kozlov V.V., The Euler-Jacobi-Lie integrability theorem, Regul. Chaotic Dyn. 18 (2013), 329-343.

[29] Llibre J., Ramírez R., Sadovskaia N., Integrability of the constrained rigid body, Nonlinear Dynam. 73 (2013), 2273-2290.

[30] Molina-Becerra M., Galán-Vioque J., Freire E., Dynamics and bifurcations of a nonholonomic Heisenberg system, Internat. J. Bifur. Chaos Appl. Sci. Engrg. 22 (2012), 1250040, 14 pages.

[31] Rosochatius E., Über die Bewegung eines Punktes, inaugural Dissertation, Univ. Göttingen, Berlin, 1877.

[32] Smirnov R.G., On the classical Bertrand-Darboux problem, J. Math. Sci. 151 (2008), 3230-3244, math-ph/0604038.

[33] Suslov G.K., Theoretical mechanics, Gostekhizdat, Moscow, 1946.

[34] Tsiganov A., Integrable Euler top and nonholonomic Chaplygin ball, J. Geom. Mech. 3 (2011), 337-362, arXiv:1002.1123.

[35] Tsiganov A.V., On the Poisson structures for the nonholonomic Chaplygin and Veselova problems, Regul. Chaotic Dyn. 17 (2012), 439-450.

[36] Tsiganov A.V., One family of conformally Hamiltonian systems, Theoret. and Math. Phys. 173 (2012), 1481-1497, arXiv:1206.5061.

[37] Tsiganov A.V., On the Lie integrability theorem for the Chaplygin ball, Regul. Chaotic Dyn. 19 (2014), 185-197, arXiv:1312.1055.

[38] Tsiganov A.V., Killing tensors with nonvanishing Haantjes torsion and integrable systems, Regul. Chaotic Dyn. 20 (2015), 463-475.

[39] Valent G., Ben Yahia H., Neumann-like integrable models, Phys. Lett. A 360 (2007), 435-438, math-ph/0512027.

[40] Veselova L.E., New cases of the integrability of the equations of motion of a rigid body in the presence of a nonholonomic constraint, in Geometry, Differential Equations and Mechanics (Moscow, 1985), Moscow State University, Moscow, 1986, 64-68.

[41] Wojciechowski S., Integrable one-particle potentials related to the Neumann system and the Jacobi problem of geodesic motion on an ellipsoid, Phys. Lett. A 107 (1985), 106-111. 